\title{
Diversidad de insectos plagas y benéficos asociados al cultivo de chayote en Matagalpa, Nicaragua 2017
}

\author{
Diversity of pests and beneficial insects associated with chayote cultivation in \\ Matagalpa, Nicaragua 2017
}

Edgardo Jiménez-Martínez ${ }^{1}$

Bayardo José González Medrano ${ }^{2}$

Ángelo Josué Centeno Leiva ${ }^{3}$

\section{Resumen}

El chayote (Sechium edule, (Jacq.) Swartz) es una hortaliza versátil; se aprovechan raíces, tallos, hojas, zarcillos, semilla, frutos y puntas de las guías. El objetivo primordial de este estudio fue identificar abundancia, riqueza, índice de diversidad, fluctuación poblacional y el hábito de las principales familias de insectos asociados al chayote. La investigación es cualitativa de tipo no experimental, fundamentada en el método descriptivo, se realizó en el período de enero a mayo del 2017 en las comunidades Molino Sur y El Caracol, municipio de Sébaco, departamento de Matagalpa, en dos fincas donde se colocaron 12 trampas para insectos, se realizó una recolección de hojas para extraer, identificar y clasificar insectos chupadores. Se identificó 65 especies de insectos y una de arácnidos pertenecientes a 46 familias de 12 órdenes, entre las principales familias destacan: Nitidulidae, Vespidae, Chrysopidae, Forficulidae, Noctuidae, Formicidae, Muscidae y Gryllidae. La diversidad insectil se estimó utilizando el índice de Simpson, la finca Sarsalosa posee más abundancia en especies por familias de insectos y la finca Las Vegas presentó la mayor diversidad. La distribución temporal de las familias más importantes fueron Nitidulidae, Vespidae, Chrysopidae, Forficulidae y Noctuidae, con polífagos, filófagos, necrófagos, rizófagos, zoófagos, nectarívoro, fitófagos, omnívoro y saprófago, como principales hábitos alimenticios.

Palabras clave: chayote; insectos; abundancia; diversidad.

\section{Summary}

Chayote (Sechium edule, (Jacq.) Swartz) is a versatile vegetable; roots, stems, leaves, tendrils, seed, fruits and tips of the guides are used. The primary objective of this study was to identify abundance, richness, diversity index, population fluctuation and habit of the main families of insects associated with chayote. The research is qualitative of a non-experimental type, based on the descriptive method, it was carried out in the

$1 \mathrm{PhD}$ en Entomología, Docente Investigador, Director de Investigación, Extensión y Posgrado, Universidad Nacional Agraria, Nicaragua. Correo: edgardo.jimenez@ci.una.edu.ni ORCID: https://orcid.org/oooo-0003-1086-7380

2 Ingeniero Agrónomo. Universidad Nacional Agraria, Nicaragua.

3 Ingeniero Agrónomo. Universidad Nacional Agraria, Nicaragua.

Recibido: 26/04/2020 Aprobado: 28/05/2020 
period from January to May 2017 in Molino Sur and El Caracol communities, Sébaco municipality, department of Matagalpa, in two farms where 12 insect traps, a leaf collection was carried out to extract, identify and classify sucking insects. 65 species of insects and one of arachnids belonging to 46 families of 12 orders were identified, among the main families are: Nitidulidae, Vespidae, Chrysopidae, Forficulidae, Noctuidae, Formicidae, Muscidae and Gryllidae. Insect diversity was estimated using the Simpson index, the Sarsalosa farm has more species abundance by insect families and Las Vegas farm presented the highest diversity. The temporal distribution of the most important families were Nitidulidae, Vespidae, Chrysopidae, Forficulidae and Noctuidae, with polyphages, phylophages, ghouls, rhizophages, zoophages, nectarivores, phytophages, omnivores and saprophages, as the main feeding habits.

Keywords: Chayote; insects; abundance; diversity.

\section{Introducción}

El cultivo del chayote (Sechium edule,( Jacq.) Swartz), tiene su origen en Mesoamérica: México y Guatemala. Las crónicas sobre la fruta se remontan a la época de la conquista, que indican que en México, el chayote ha sido cultivado para su consumo antes de la colonizacion y que entre el sur de Mexico y Guatemala se concentra la variabilidad genética, en donde inició su distribución hacia el sur. De acuerdo a otro tipo de evidencia, la procedencia de su nombre proviene del náhualt que significa "calabaza espinoza" dando más veracidad que su origen sea México (PRODES, 2001). La presente investigación se realizó con el fin de identificar y describir los principales insectos que están asociados con el cultivo de chayote, para determinar la diversidad, fluctuación, taxonomía, dinámica e incidencia poblacional de los insectos. Se pretende generar nuevos conocimientos y contribuir a desarrollar estrategias de manejo integrado de plagas que puedan ser utilizadas por productores en las plantaciones de chayote en Nicaragua.

Según Orozco (1998), la composición en 100 gramos de materia comestible de la fruta del chayote es de 31.0 de caloría, $98.8 \%$ agua, $0.9 \mathrm{~g}$ de proteína, $7.7 \mathrm{~g}$ de carbohidratos, o.6 g de fibra, $12.0 \mathrm{mg}$ de calcio, o.6 mg de hierro, vitamina A 5.o U.I, vitamina B2 $0.03 \mathrm{mg}$ y vitamina $C 20.0 \mathrm{mg}$. En Nicaragua esta planta ha formado parte de los hogares destinada para autoconsumo. En el norte del país se han observado un gran número de genotipos de chayote, en Somoto se puede encontrar gran diversidad con diferentes características como forma, color, presencia de espinas, tamaño del fruto, color de hojas, entre otras características fenotípicas interesantes, aunque este acervo genético no ha sido aprovechado a nivel comercial (INTA, 2004).

Actualmente las zonas de mayor producción del cultivo del chayote son: El Hatillo, Jamaica, Aguas Frías, San Miguel, El molino, La labranza, Jumaiki, ubicados al este del municipio de Sébaco del departamento de Matagalpa, siendo los límites territoriales al 
norte con Jinotega, al sur con Terrabona y Darío, al este con el municipio de Matagalpa y al oeste con Río Viejo (Reyes, 2003).

En cuanto a las principales plagas que se pueden hacer mención se encuentran los ácaros, siendo (Tetranychus urticae K.) la especie que afecta a esta cucurbitácea, trips asociados con el chayote que hasta el momento se ha clasificado como (Frankliniella Williamsi H.) (Retana, 2002) y el gusano perforador del fruto (Diaphania nitidalis S.) en el cual su estadio larval afecta las yemas, flores, tallos, hojas y perforaciones en el fruto; en las frutas dañadas se observan excrementos en masas pequeñas de color pardo (Corrales, 1980).

En Nicaragua, los productores de chayote desconocen la diversidad y fluctuación de los insectos que están presentes en este cultivo, por lo tanto no saben con certeza la relación entre insectos benéficos e insectos plagas y por consiguiente se encuentran con una serie de problemas al momento de realizar el manejo, de igual modo la falta de documentos de régimen científico y la poca información nacional con reportes de los principales órdenes, familias, géneros y especies de insectos asociados a este rubro.

\section{Materiales y métodos}

\section{Ubicación del estudio}

El estudio se realizó de enero a mayo del 2017, en dos unidades de producción con parcelas de chayote. Las fincas están ubicadas en las comunidades Molino Sur y El Caracol, municipio de Sébaco, departamento de Matagalpa, con una extensión territorial de $291 \mathrm{~km}^{2}$ a una altitud de $613 \mathrm{msnm}$ en las coordenadas $12^{\circ} 50^{\prime} 5.16^{\prime \prime}$ latitud Norte y $85^{\circ} 59^{\prime} 41.74^{\prime \prime}$ longitud Oeste, la precipitación anual varía entre los 800 y 2000 mm (MAG, 2017).

Durante el período de muestreo, en la zona se registraron $287.2 \mathrm{~mm}$ de precipitación, en su mayor parte distribuido en el mes de mayo, mientras que la temperatura promedio fue de $26.28^{\circ} \mathrm{C}$. Con relación al año anterior la temperatura promedio fue de $26.09^{\circ} \mathrm{C}$, mientras que la precipitación de $791.9 \mathrm{~mm}$ y las precipitaciones fueron inferiores debido a las sequias (INTA, 2017).

La finca Sarsalosa está ubicada en la comunidad El Caracol propiedad de la Sra. María de la Cruz Velásquez con una parcela de chayote de $7026 \mathrm{~m}^{2}$ con cinco meses de establecido, manejado de forma convencional; se encuentra a una altitud de 667 msnm, en las coordenadas X: 60097 '63" y Y: 140 17' 767".

La finca Las Vegas está ubicada en la comunidad Molino Sur, propiedad del Sr. Alcides Leiva con una parcela de chayote de $3513 \mathrm{~m}^{2}$, con cinco meses de establecido 
y manejada de forma convencional, se encuentra a una altitud de $613 \mathrm{msnm}$ en las coordenadas X: 600 97' 32" y Y: 140 18' 78"2.

\section{Diseño del estudio}

Se estableció un modelo de investigación cualitativa no experimental que se fundamenta en el método descriptivo, forma de investigación aplicada en donde no hay manipulación de variables (UNA, 2008). Consistió en la comparación de dos fincas de chayote ubicadas en el municipio de Sébaco, comunidad Molino Sur y El Caracol, la cosecha del cultivo se realizó cada ocho días, al igual que la aplicación de insecticidas. En cada finca se colocaron 12 trampas: seis de caída libre y seis de galón con melaza a una altura de dos metros sobre el suelo.

La colecta de insectos se realizó semanalmente en ambas fincas, utilizando dos métodos de trampeo.

\section{Descripción del primer método de captura de insectos}

Para la captura y colecta de insectos rastreros y algunos voladores se utilizaron trampas de caída libre en el suelo, que consistió en colocar panas plásticas de color amarilla en un hueco del mismo diámetro de las panas, las dimensiones de las panas fueron de $30 \mathrm{~cm}$ de diámetro y $15 \mathrm{~cm}$ de profundidad con capacidad de cuatro litros de agua, a estas se le agregó 20 g de detergente xedex ${ }^{\circledR}$ en dos litros de agua, la frecuencia de colecta fue semanal y en cada trampa se sustituyó la solución de agua y detergente después de haber realizado el muestreo.

\section{Descripción del segundo método de captura de insectos}

Para la captura y colecta de insectos voladores se utilizaron trampas de galones plásticos de color azul con capacidad de 3.78 litros de agua y se le agregarán 20 cc de melaza como un atrayente para los insectos voladores y un litro de agua, los galones fueron cortados por los dos costados arriba de la mitad y colgados al alambre verticalmente a una altura de 2 metros. La frecuencia de colecta fue semanalmente, en cada fecha de colecta se cambió la solución del agua y melaza.

\section{Procesamiento de muestras e identificación de insectos a nivel de laboratorio}

El procesamiento de las muestras de insectos en el laboratorio, consistió inicialmente en sacar el espécimen de los vasos colectores por fecha y por finca, estos se vaciaron individualmente sobre papel absorbente, posteriormente con la ayuda de pinceles finos, se realizó la separación de los insectos capturados, para el montaje de los especímenes se utilizaron alfileres entomológicos (MORPHO de $4 \mathrm{~cm}$ ), para 
la identificación de los especímenes se utilizó estereoscopios (CARL ZEISS, modelo 475002).

La identificación se realizó a nivel de orden, familia y especie, esta se realizó en el laboratorio de entomología de la UNA; para la identificación de familias se utilizaron claves taxonómicas dicotómicas propuestas por Nunes y Dávila (2004). Se hizo la identificación hasta el taxón de género y especie, para ello se enviaron los especímenes al profesor Alex Cerrato responsable del museo entomológico de la UNA, quien, utilizó claves dicotómicas morfológicas. Se consultó la literatura de las principales familias y subfamilias de insectos de interés agrícola en Nicaragua (Andrews y Caballero, 1989), Texto Básico: Entomología (Jiménez, 2009), Insectos de Nicaragua: catálogo de los insectos y artrópodos terrestres de Nicaragua (Maes, 1998) y el texto de Entomología sistemática (Sáenz y De La Llana, 1990).

\section{Variables evaluadas en el estudio}

\section{Descripción taxonómica y hábitos alimenticios de las especies insectiles}

Se organizó en un cuadro donde se muestra la información de todos los insectos encontrados en este estudio.

\section{Poblaciones insectiles fitófagas y benéficas encontradas en el cultivo de chayote}

Se realizó revisión de literatura de acuerdo a los insectos encontrados para determinar el comportamiento (plagas o benéficos) de cada población de insectos.

\section{Comparación de la abundancia de insectos de los principales órdenes por finca}

Se hizo sumatoria de los principales órdenes de insectos encontrados en todas las fechas de muestreo en las fincas Sarsalosa y Las Vegas.

\section{Comparación de la abundancia total de insectos por familia encontrados por finca}

Se hizo un conteo de la abundancia de insectos de las diferentes familias encontradas en todas las fechas de colectas en las fincas Sarsalosa y Las Vegas, para determinar cuál era la finca con mayor número de familias encontradas.

\section{Riqueza total de familias de insectos encontrados por finca}

Se hizo un conteo del total de insectos por familias encontradas en todas las fechas de colectas en las fincas Sarsalosa y Las Vegas, para determinar cuál era la finca con mayor riqueza de familias insectiles. 


\section{Fluctuación poblacional de la familia Nitidulidae por finca}

Se comparó la distribución temporal de insectos de la familia Nitidulidae por fechas de colecta, donde se hizo la sumatoria del número de insectos encontrados en las fincas Sarsalosa y Las Vegas.

\section{Fluctuación poblacional de la familia Vespidae por finca}

Se comparó la distribución temporal de insectos de la familia Vespidae por fechas de colecta, donde se hizo la sumatoria del número insectos encontrados en las fincas Sarsalosa y Las Vegas.

\section{Fluctuación poblacional de la familia Chrysopidae por finca}

Se comparó la distribución temporal de insectos de la familia Chrysopidae por fechas de colecta, donde se hizo la sumatoria del número insectos encontrados, en las fincas Sarsalosa y Las Vegas.

\section{Análisis de los datos}

Después de colectados los insectos en el campo, se procedió a ingresar los datos por familias en una hoja de Excel, se procedió a ordenarlos de mayor a menor, se realizó la separación por finca y por tipo de trampa, utilizando los mismos datos se agrupó por órdenes y familias de la más abundante a la menos abundante.

\section{Resultados y discusión}

Descripción taxonómica y hábitos alimenticios de las especies insectiles encontrados en el cultivo de chayote.

En el Cuadro 1, se presentan 12 órdenes de insectos encontrados en el estudio, entre ellos Coleóptera, Hymenoptera, Hemíptera, Díptera, Orthoptera, Odonata, Dermáptera, Mantodea, Blattodea, Lepidóptera, Neuróptera, Thysanoptera, además del orden Trombidiforme perteneciente a la clase arácnida, se presenta el hábito alimenticio de cada orden de los insectos encontrados en la finca Sarsalosa y Las Vegas, los cuales se clasifican en polífagos, filófagos, necrófagos, rizófagos, zoófagos, nectarívoro, fitófagos, omnívoro y saprófagos. 
Cuadro 1: Descripción taxonómica y hábitos alimenticios de las especies insectiles encontrados en el cultivo de chayote

\begin{tabular}{|c|c|c|c|c|c|}
\hline Orden & Familia & Género & Especie & Nombre común & Hábito Alimenticio \\
\hline \multirow[t]{23}{*}{ Coleóptero } & Scarabaeidae & Pedilonota & Sp & Escarabajo & Polífagos \\
\hline & Scarabaeidae & Euphoria & $\mathrm{Sp}$ & Escarabajo & Polífagos \\
\hline & Scarabaeidae & Heterogonphus & $\mathrm{Sp}$ & Escarabajo & Polífagos \\
\hline & Scarabaeidae & Anomala & $\mathrm{Sp}$ & Escarabajo & Polífagos \\
\hline & Carabidae & Colosoma & $\mathrm{Sp}$ & Escarabajo & Polífagos \\
\hline & Carabidae & Colleidae & $\mathrm{Sp}$ & Escarabajo & Polífagos \\
\hline & Chrysomelidae & Colligrapha & $\mathrm{Sp}$ & Vaquita & Filófagos \\
\hline & Silphidae & Silpha & $\mathrm{Sp}$ & Escarabajo & Necrófagos \\
\hline & Curculionidae & Cosmopolistes & $\mathrm{Sp}$ & Picudo & Polífagos \\
\hline & Cerambycidae & Aneflus & $\mathrm{Sp}$ & Escarabajo & Filófagos \\
\hline & Cerambycidae & Logocherius & Sp & Escarabajo & Filófagos \\
\hline & Cerambycidae & Cylene & $\mathrm{Sp}$ & Escarabajo & Filófagos \\
\hline & Cerambycidae & Trox & $\mathrm{Sp}$ & Escarabajo & Filófagos \\
\hline & Meloidae & Pyrota & $\mathrm{sp}$ & Escarabajo & Polífagos \\
\hline & Tenebrionidae & Zoperus & $\mathrm{sp}$ & Escarabajo & Polífagos \\
\hline & Tenebrionidae & Colephorus & Sp & Escarabajo & Polífagos \\
\hline & Tenebrionidae & Epitragus & Sp & Escarabajo & Polífagos \\
\hline & Tenebrionidae & Rhinondrus & $\mathrm{sp}$ & Escarabajo & Polífagos \\
\hline & Lycidae & Calopteron & $\mathrm{Sp}$ & Escarabajo & Polífagos \\
\hline & Mordellidae & Mordellistena & $\mathrm{Sp}$ & Escarabajo & Polífagos \\
\hline & Nitidulidae & Carpophilus & $\mathrm{Sp}$ & Escarabajo & Polífagos \\
\hline & Elateridae & Conoderus & $\mathrm{Sp}$ & Escarabajo & Rizófagos \\
\hline & $\begin{array}{l}\text { Coccinellidae } \\
\text { Coccinellidae }\end{array}$ & $\begin{array}{l}\text { Hypodamia } \\
\text { Cycloneda }\end{array}$ & $\begin{array}{c}\text { Sp } \\
\text { sanguínea }\end{array}$ & $\begin{array}{l}\text { Mariquita } \\
\text { Mariquita }\end{array}$ & $\begin{array}{l}\text { Zoófagos } \\
\text { Zoófagos }\end{array}$ \\
\hline \multirow[t]{9}{*}{ Hymenoptera } & Pompilidae & Pesis & mexicana & Avispa & Nectarivoros \\
\hline & Colletidae & Colletes & $\mathrm{Sp}$ & Avispa & Nectarivoros \\
\hline & Pompilidae & Pepsis & $\mathrm{Sp}$ & Avispa & Nectarivoros \\
\hline & Vespidae & Polistes & $\mathrm{Sp}$ & Catala & Zoófagos \\
\hline & Vespidae & Polybia & instabilis & Avispa & Nectarivoros \\
\hline & Vespidae & Polistes & Exclamans & Catala & Polífagos \\
\hline & Vespidae & Polybia & $\mathrm{sp}$ & Avispa & Zoófagos \\
\hline & Eumeridae & Eumenes & $\mathrm{sp}$ & Avispa alfarera & Nectarivoro \\
\hline & Formicidae & Atta & $\mathrm{sp}$ & Zompopo & Fitófagos \\
\hline
\end{tabular}




\begin{tabular}{|c|c|c|c|c|c|}
\hline Orden & Familia & Género & Especie & Nombre común & Hábito Alimenticio \\
\hline & Formicidae & Camponotus & Chilensis & Hormigon & Omnívoros \\
\hline & Formicidae & Solenopsis & Sp & Hormiga & Zoófagos \\
\hline & Anthoporidae & Xylocopa & Sp & Abejorro negro & Nectarivoros \\
\hline & Halictidae & Halictus & $\mathrm{Sp}$ & Abejita & Nectarivoros \\
\hline & Apidae & Apis & Mellifera & Abeja & Nectarivoros \\
\hline & Apidae & Trigona & Sp & Congo negro & Nectarivoros \\
\hline \multirow[t]{10}{*}{ Hemípteros } & Cydnidae & Pongaeus & $\mathrm{Sp}$ & Chinche & Rizófagos \\
\hline & Pentatomidae & Euchistas & $\mathrm{Sp}$ & $\begin{array}{c}\text { Chinche ma- } \\
\text { rrón }\end{array}$ & Fitófagos \\
\hline & Pentatomidae & Euchistas & bifimulus & $\begin{array}{c}\text { Chinche ma- } \\
\text { rrón }\end{array}$ & Fitófagos \\
\hline & Pentatomidae & Proxis & punctulatus & Chinche loco & Polífagos \\
\hline & Pentatomidae & Loxa & viridis & Chinche verde & Polífagos \\
\hline & Cicadellidae & Oncomotopia & $\mathrm{Sp}$ & Chicharria & Fitófagos \\
\hline & Cicadellidae & Sibobia & $\mathrm{Sp}$ & Chicharra & Fitófagos \\
\hline & $\begin{array}{l}\text { Cicadidae } \\
\text { Coccidae }\end{array}$ & $\begin{array}{c}\text { Diceroprocta } \\
\text { Ceroplastes }\end{array}$ & $\begin{array}{l}\mathrm{Sp} \\
\mathrm{Sp}\end{array}$ & $\begin{array}{l}\text { Chicharra } \\
\text { Tortuguilla }\end{array}$ & $\begin{array}{l}\text { Polífagos } \\
\text { Fitofagos }\end{array}$ \\
\hline & Coreidae & Mozena & $\mathrm{Sp}$ & Chinche & Fitófagos \\
\hline & $\begin{array}{l}\text { Reduviidae } \\
\text { Aphididae }\end{array}$ & $\begin{array}{c}\text { Apiomeris } \\
\text { Aphis }\end{array}$ & $\begin{array}{l}\mathrm{Sp} \\
\mathrm{Sp}\end{array}$ & $\begin{array}{l}\text { Chinche } \\
\text { Asesino } \\
\text { Afidos }\end{array}$ & $\begin{array}{l}\text { Zoófagos } \\
\text { Fitófagos }\end{array}$ \\
\hline \multirow[t]{7}{*}{ Dípteros } & Stratiomidae & Hermetia & Illucen & Mosca soldado & Saprófagos \\
\hline & Tachinidae & Lespesia & Archivora & Mosca & Saprófagos \\
\hline & Tachinidae & Lespesia & Sp & Mosca & Saprófagos \\
\hline & Sarcophagidae & Sarcophaga & $\mathrm{Sp}$ & Mosca & Saprófagos \\
\hline & Muscidae & Musa & Domestica & Mosca común & Saprófagos \\
\hline & $\begin{array}{l}\text { Syrphidae } \\
\text { Syrphidae }\end{array}$ & $\begin{array}{c}\text { Allograpta } \\
\text { Bacha }\end{array}$ & $\begin{array}{c}\text { Sp } \\
\text { clavata }\end{array}$ & $\begin{array}{l}\text { Mosca } \\
\text { Mosca }\end{array}$ & $\begin{array}{c}\text { Nectarivoros } \\
\text { Saprofagos }\end{array}$ \\
\hline & Calliphoridae & Phaenecia & Sericata & Mosca & Saprófagos \\
\hline \multirow[t]{3}{*}{ Orthoptera } & Tettigoniidae & Neocepholus & $\mathrm{Sp}$ & Ezperanza & Fitófagos \\
\hline & Romaleidae & Taeniopoda & $\mathrm{Sp}$ & Chapulin & Fitófagos \\
\hline & Gryllidae & Acheta & Similis & Grillo & Fitófagos \\
\hline \multirow[t]{3}{*}{ Odonata } & Coenagrionidae & Coenagrion & $\mathrm{Sp}$ & Pipilacha & Zoófagos \\
\hline & Calopterygidae & Libellula & $\mathrm{Sp}$ & Pipilacha & Zoófagos \\
\hline & Calopterygidae & Libellula & $\mathrm{Sp}$ & Libelula & Zoófagos \\
\hline Dermaptera & Forficulidae & Dorus & linearis & Tijereta & Zoófagos \\
\hline
\end{tabular}


AGROPECUARIO

\begin{tabular}{|c|c|c|c|c|c|}
\hline Orden & Familia & Género & Especie & Nombre común & Hábito Alimenticio \\
\hline Mantodea & Mantidae & Mantis & $\mathrm{Sp}$ & Madre culebra & Zoófagos \\
\hline Blattodea & Blattellidae & Blattella & germonica & Cucaracha & Saprófagos \\
\hline Lepidóptera & Noctuidae & Spodoptera & $\mathrm{Sp}$ & Palomilla & Nectarivoros \\
\hline & Noctuidae & Spodoptera & Frujiperda & Cogollero & Fitófagos \\
\hline & Papilionidae & Battus & Polydamas & Mariposa & Fitófagos \\
\hline Thysanoptera & Thripidae & Thrips & Tabaci & Trips & Fitofagos \\
\hline Neuróptera & Chrysopidae & chrisopa & $\mathrm{Sp}$ & Leon de afidos & Zoófagos \\
\hline Trombidiforme & Tetranychidae & Tetranychus & $\mathrm{Sp}$ & Araña roja & Fitófago \\
\hline
\end{tabular}

\section{Poblaciones insectiles fitófagas y benéficas}

Se encontró que el número total de insectos fitófagos fue mayor en la finca Sarsalosa con 1341, en comparación con la finca Las Vegas que presentó 1 180. En el recuento de los insectos benéficos, se encontró un mayor número de insectos en la finca Sarsalosa con 1422 , comparado con la finca Las vegas con 987 insectos benéficos en total (Figura 1).

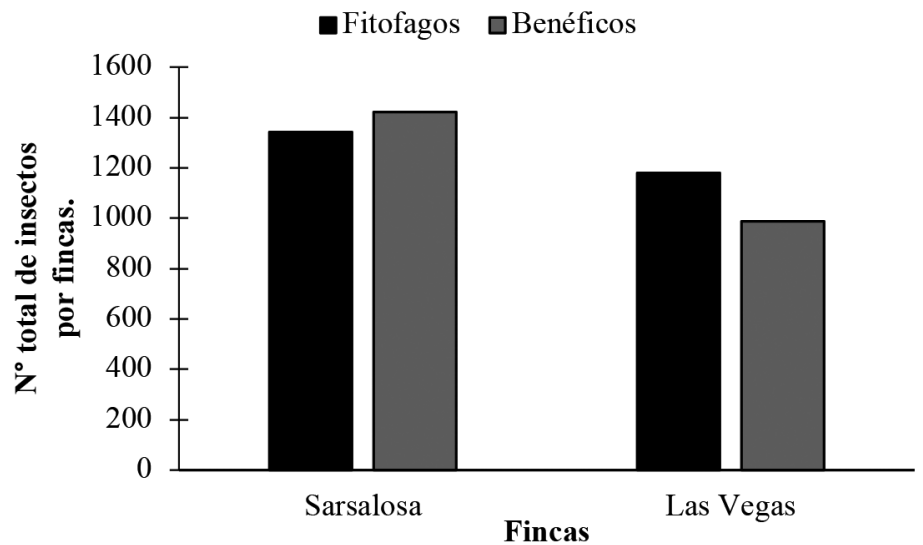

Figura No. 1: Poblaciones insectiles fitófagas y benéficas encontradas en el cultivo de chayote.

\section{Comparación y abundancia total de los órdenes de insectos}

Se encontró mayor cantidad de insectos por orden en la finca Sarsalosa con la presencia de los 13 órdenes con respecto a la finca Las Vegas, en la cual se presentaron 12 órdenes (Cuadro 2). En la finca Sarsalosa los órdenes Coleópteros, Hymenoptera, Díptera, Neuróptera, Lepidóptera, Blattodea y Mantodea fueron más abundantes, mientras que en la finca Las Vegas los órdenes dermápteros, hemíptero, orthoptera y odonata fueron más abundantes en comparación con la aparición que tuvieron en la finca Sarsalosa. 
Los órdenes Thysanoptera y Trombidiforme fueron encontrados a través del muestreo directo en el cual no se especifica su abundancia.

Según Andrews y Quezada (1989), en el campo las poblaciones fluctúan en el tiempo, cuando los procesos aditivos tienen un impacto más grande que la fuerza sustractiva, la densidad poblacional aumenta. Los insectos del orden hymenoptera poseen muchas especies benéficas debido a que intervienen en la polinización de las plantas, otros son considerados agentes de control natural y biológico de plagas y malezas; otros producen cera y miel y otras especies son importantes porque pican al hombre y a otros animales (Sáenz y De La Llana, 1990). Las especies del orden neuróptera, conocidas comúnmente con el nombre de crisopas u hormigas león, tienen gran importancia en la agricultura porque son depredadores de insectos fitófagos, en muchas especies la coloración de los adultos es parda o verde, aunque algunas son de colores fuertes y vistosos (Jiménez, 2009).

Cuadro No. 2: Comparación y abundancia total de los órdenes de insectos encontrados en el cultivo de chayote

\begin{tabular}{|l|l|l|l|}
\hline \multirow{2}{*}{ Ordenes de insectos. } & \multicolumn{3}{c|}{ Número de insecto por ordenes } \\
\cline { 2 - 4 } & \multicolumn{2}{|c|}{ Sarsalosa } & \multicolumn{2}{c|}{ Las Vegas } & \multicolumn{1}{c|}{ Total } \\
\hline Coleópteros & 875 & 544 & 1419 \\
\hline Hymenoptera & 634 & 420 & 1054 \\
\hline Díptera & 300 & 272 & 572 \\
\hline Neuróptera & 369 & 56 & 425 \\
\hline Lepidoptera & 193 & 182 & 375 \\
\hline Dermaptera & 65 & 304 & 369 \\
\hline Hemiptera & 160 & 161 & 321 \\
\hline Orthoptera & 91 & 156 & 247 \\
\hline Odonata & 23 & 63 & 86 \\
\hline Blattodea & 50 & 9 & 59 \\
\hline Mantodea & 3 & 0 & 3 \\
\hline Total & 2763 & 2167 & 4930 \\
\hline
\end{tabular}

\section{Comparación y abundancia total de familia de insectos}

Se encontró mayor cantidad de familias en la finca Sarsalosa, con un total de 45 con respecto a 37 en la finca Las Vegas, de 46 familias registradas y clasificadas (Cuadro 3). La familia más abundante fue Nitidulidae, con mayor presencia en la finca Sarsalosa con 357 especimenes con respecto a 135 encontradas en la finca Las Vegas, estos insectos son primariamente saprófagos, es decir, se alimentan de plantas, frutos podridos, miel, polen, animales muertos, carroña y estiércol (Neumann y Elzen, 2004). 
La familia Vespidae fue la segunda más abundante, en la finca Sarsalosa se obtuvo mayor número de insectos de la familia Vespidae con 329 especímenes recolectados, en comparación de 129 recolectados en la finca Las Vegas. Los insectos de la familia Chrysopidae, son los más importantes del orden Neuróptera, son considerados los agentes biológicos decisivos en el control de plagas insectiles, (Gómez, 2011). Durante el período de muestreo y colecta se hizo un recuento de 425 especímenes distribuidos entre la finca Sarsalosa con 369 y Las Vegas con 56.

Montano y Bustamante (2016) encontraron una abundancia total de insectos de las familias Vespidae de 203, Noctuidae 529 y Apidae 235 en trampas de caída libre y galones, en cambio en el cultivo de chayote se encontró una abundancia de insectos de la familia Vespidae de 458, Noctuidae de 305 y Apidae de 186.

Cuadro No. 3: Comparación y abundancia total familias de insectos y ácaros encontrados en el cultivo de chayote

\begin{tabular}{|l|l|l|l|}
\hline \multirow{2}{*}{ Familias } & \multicolumn{2}{|c|}{ Especimenes capturados por finca } & \multirow{2}{*}{ Total } \\
\cline { 2 - 3 } & \multicolumn{2}{|c|}{ Sarsalosa } & \multicolumn{2}{c|}{ Las Vegas } & \multicolumn{1}{c|}{} \\
\hline Nitidulidae & 357 & 135 & 492 \\
\hline Vespidae & 329 & 129 & 458 \\
\hline Chrysopidae & 369 & 56 & 425 \\
\hline Forficulidae & 65 & 304 & 369 \\
\hline Noctuidae & 157 & 148 & 305 \\
\hline Formicidae & 131 & 166 & 297 \\
\hline Muscidae & 132 & 116 & 248 \\
\hline Gryllidae & 61 & 151 & 212 \\
\hline Curculionidae & 14 & 195 & 209 \\
\hline Apidae & 113 & 73 & 186 \\
\hline Scarabaeidae & 132 & 33 & 165 \\
\hline Tachinidae & 31 & 113 & 144 \\
\hline Tenebrionidae & 40 & 78 & 118 \\
\hline Pentatomidae & 11 & 106 & 117 \\
\hline Cicadellidae & 76 & 24 & 100 \\
\hline Sarcophagidae & 65 & 17 & 82 \\
\hline Calliporidae & 56 & 24 & 80 \\
\hline Elateridae & 55 & 21 & 76 \\
\hline Carabeidae & 34 & 40 & 71 \\
\hline Papilionidae & 50 & 9 & 59 \\
\hline Blattelidae & & & 70 \\
\hline
\end{tabular}




\begin{tabular}{|c|c|c|c|}
\hline \multirow{2}{*}{ Familias } & \multicolumn{2}{|c|}{ Especimenes capturados por finca } & \multirow{2}{*}{ Total } \\
\hline & Sarsalosa & Las Vegas & \\
\hline Coccinellidae & 48 & 9 & 57 \\
\hline Coenagrionidae & 0 & 57 & 57 \\
\hline Lycidae & 54 & 0 & 54 \\
\hline Silphidae & 51 & 0 & 51 \\
\hline Mordellidae & 50 & 0 & 50 \\
\hline Chrysomelidae & 14 & 29 & 43 \\
\hline Pompilidae & 23 & 19 & 42 \\
\hline Cicadidae & 8 & 28 & 36 \\
\hline Halictidae & 16 & 18 & 34 \\
\hline Calopterygidae & 23 & 6 & 29 \\
\hline Reduviidae & 29 & 0 & 29 \\
\hline Cydnidae & 24 & 3 & 27 \\
\hline Eumenidae & 12 & 15 & 27 \\
\hline Cerambicidae & 26 & 0 & 26 \\
\hline Stratiomidae & 16 & 2 & 18 \\
\hline Tettigonidae & 13 & 5 & 18 \\
\hline Romaleidae & 17 & 0 & 17 \\
\hline Coreidae & 12 & $\mathrm{o}$ & 12 \\
\hline Collectidae & 10 & 0 & 10 \\
\hline Meloidae & 3 & 4 & 7 \\
\hline Mantidae & 3 & $\mathrm{o}$ & 3 \\
\hline
\end{tabular}

\section{Riqueza total de familias de insectos encontradas}

La riqueza total de familias encontradas en el cultivo de chayote entre ambas fincas fue de 46, siendo la finca Sarsalosa la que mayor riqueza de familias presento con 45, comparada con la finca Las Vegas con 37 familias encontradas (Figura 2).

En un estudio realizado en el cultivo de piña por Mairena (2015) se encontró en un total de seis, riqueza total de 51 familias de insectos. Téllez y Jirón (2013) realizaron un estudio en el cultivo de marango utilizando trampas de caída libre y galones, encontrando una riqueza total de insectos de 23 familias incluyendo una de arácnidos. En el cultivo de marañón se encontró una riqueza total de 11 familias de insectos, en trampas de caída libre y galones. 


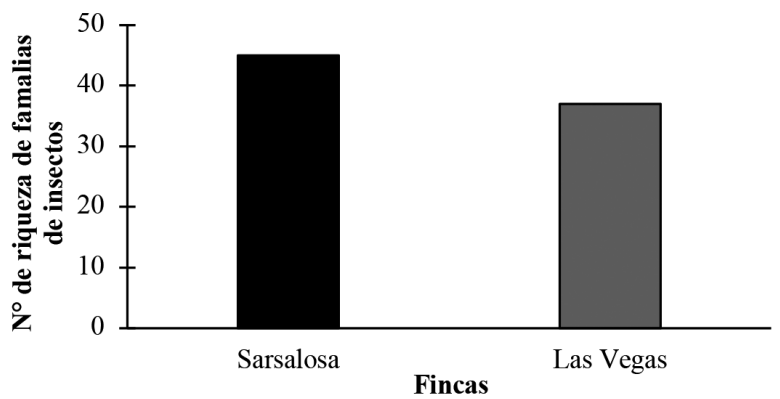

Figura No. 2: Riqueza total de familias de insectos encontrados en el cultivo de chayote en las fincas Sarsalosa y Las Vegas, en Sébaco, Matagalpa entre enero y mayo del 2017.

\section{Fluctuación poblacional de la familia Nitidulidae en el cultivo de chayote}

En la Figura 3, se muestra que las poblaciones de la familia Nitidulidae se presentaron a partir de la fecha de colecta, del 6 de enero al 12 de mayo. Los mayores picos poblacionales de esta familia se presentaron en la finca Sarsalosa, en las fechas de muestreo; 27 de enero con 35 insectos, 10 de marzo con 32 insectos y el 7 de abril con 36 insectos. En la finca Las vegas la presencia de estos insectos fue menor, presentando los mayores picos poblacionales el 17 de febrero con 18 insectos, 24 de febrero con 22 insectos y 28 de abril con 35 insectos, no presentándose en 12 ocasiones durante las 21 fechas de muestreo.

Los Nitidulidae es una familia muy variable en cuanto a forma del cuerpo, tamaño y valoración, la mayoría son pequeños, muchas veces son ovalados o alargados, algunos presentan alas cortas o muy cortas, dejando ver los últimos segmentos del abdomen. Las antenas presentan una maza de tres segmentos, la mayoría de las especies se encuentran en el material vegetal en descomposición, es decir que son saprofitos y se alimentan de jugos fermentados. Algunos se encuentran sobre hongos o sobre cadáveres de animales; algunas especies se encuentran en flores (Maes, 1998).

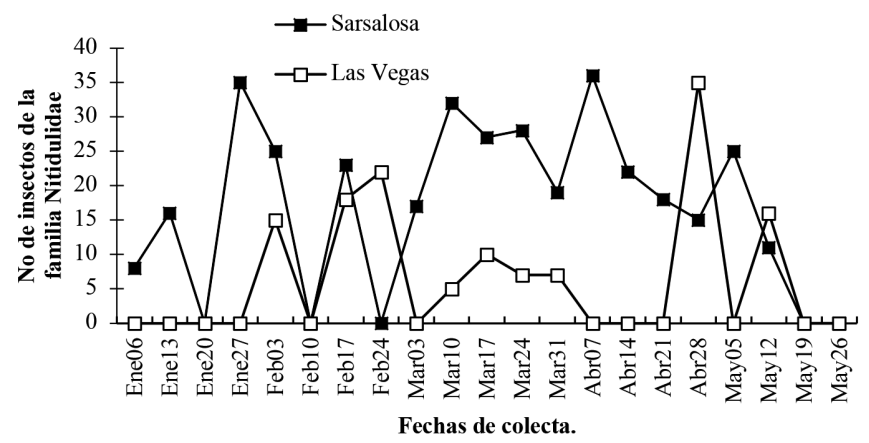

Figura No. 3: Fluctuación poblacional de la familia Nitidulidae en el cultivo de chayote en las fincas Sarsalosa y Las Vegas evaluadas entre los meses de enero a mayo del 2017. 


\section{Fluctuación poblacional de la familia Vespidae en el cultivo de chayote}

Se muestra en la Figura 4, los resultados donde la finca Sarsalosa fue la que presentó mayor número de insectos, con un total de 329 no presentándose en dos ocasiones de las 21 fechas de colecta, los mayores picos poblacionales se encontraron el 31 de marzo con 39 insectos y el 5 de mayo con 36 insectos. En la finca Las Vegas se encontraron un total de 129 insectos obteniendo su mayor pico el 13 de enero con 23 insectos y el 5 de mayo con 18 insectos colectados.

La fluctuación poblacional de la familia Vespidae comúnmente llamados avispas son comunes tanto en bosques como en campos cultivados, la familia Vespidae llama mucho la atención para los agrónomos y productores agrícolas ya que muchas especies son depredadoras en el campo (Maes,1998).

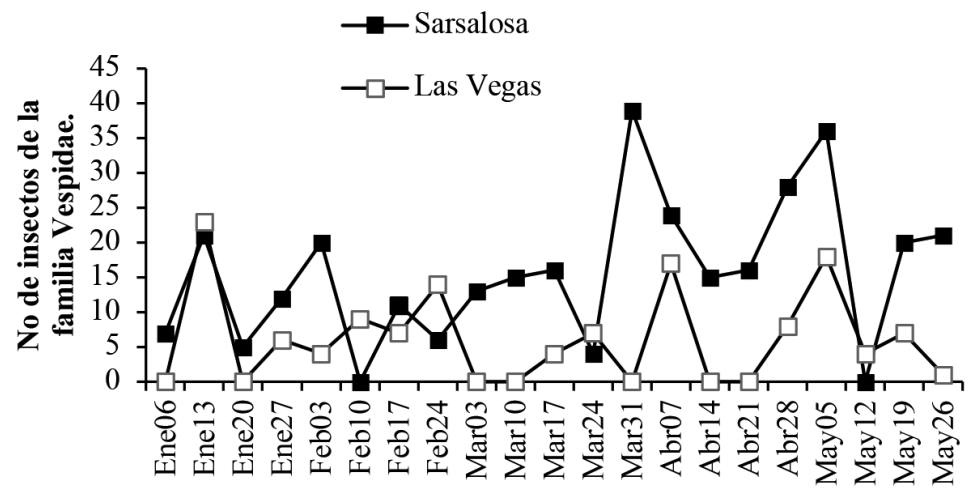

Fechas de colecta.

Figura No. 4: Fluctuación poblacional de la familia Vespidae en el cultivo de chayote en las fincas Sarsalosa y Las Vegas evaluadas entre los meses de enero a mayo del 2017.

\section{Fluctuación poblacional de la familia Chrysopidae en el cultivo de chayote}

En la Figura 5, se muestra que la mayor población de insectos fue en la finca Sarsalosa con un total de 369, no presentándose en una fecha de las 21 colectadas, obteniendo su mayor pico el 14 de abril con 33 insectos, el 10 y el 17 de marzo junto con el 07 y 21 de abril con 31 insectos colectados. En la finca Las Vegas se presentó en siete ocasiones de las 21 fechas colectadas, encontrándose un total de 56 insectos, obteniendo su pico más alto el 5 de mayo con 15 insectos colectados.

Los Chrysopidae es conocida como león de áfidos, mosca de ojos de oro o crisopa, son verdes, de alas transparentes y de ojos brillantes metálicos. Las larvas son depredadores muy útiles en los cultivos, son conocidos como depredadores de áfidos, pero también atacan huevos de muchos insectos, larvas pequeñas de lepidópteras, entre otras acciones de control biológico natural. (Maes, 1998). Los insectos de la familia 
Chrysopidae, son los más importante del orden Neuróptera, considerados los agentes biológicos decisivos para el control de plagas insectiles, (Gómez, 2011).

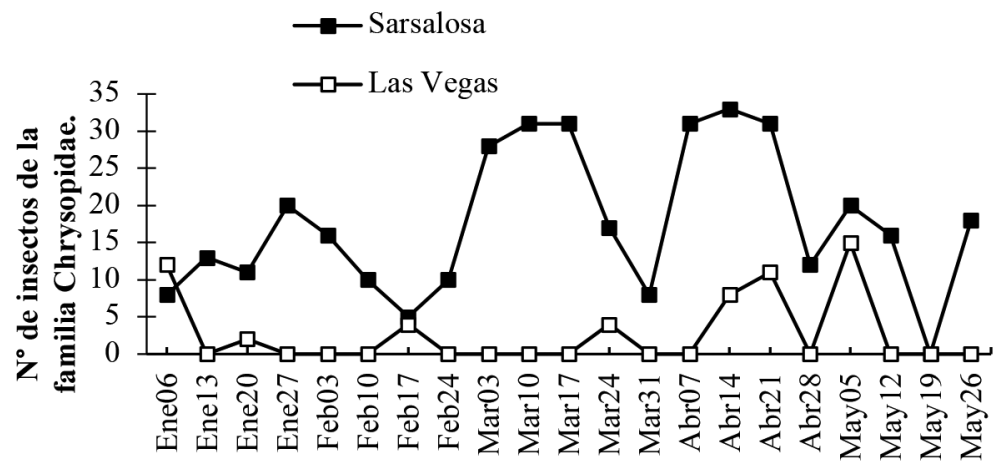

Fecha de colecta.

Figura No. 5: Fluctuación poblacional de la familia Chrysopidae en el cultivo de chayote en las fincas Sarsalosa y Las Vegas evaluadas entre los meses de enero a mayo del 2017.

\section{Conclusiones}

Se encontró un total de 66 familias de insectos, las familias más dominantes fueron la Nitidulidae, Vespidae, Chrysopidae, Forficulidae, Noctuidae particularmente en la finca Sarsalosa, a excepción la familia Forficulidae que presento mayor dominancia en la finca Las Vegas. Los mayores picos poblacionales para las familias Nitidulidae, Vespidae, Chrysopidae, fueron los meses de marzo y abril.

\section{Agradecimiento}

Esta publicación obtuvo el financiamiento de: El Fondo de Asistencia Internacional de los Estudiantes y Académicos Noruegos (SAIH).

\section{Lista de referencia}

Andrews, K. L., y Caballero, R. (1989). Guía para el estudio de órdenes y familias de insectos de Centroamérica. Francisco Morazán, Honduras: Escuela Agrícola Panamericana El Zamorano.

Andrews, K. L., y Quezada, J. R. (1989). Manejo integrado de plagas insectiles en la agricultura; estado actual y futuro. Francisco Morazán, Honduras: Escuela Agrícola Panamericana El Zamorano. 
Corrales, G. (1980). Principales plagas de las Hortalizas. Escuela de ciencias Agropecuarias Universidad Nacional, Heredia, Costa Rica.

Gómez Martínez. J. (2011). Entomofauna y patógenos asociados al cultivo de marañón (Anacardium occidentale L.), en León, Nicaragua, entre los meses de julio 2009 a marzo 2010. (Tesis de maestría). Recuperada de http://repositorio.una.edu. ni/2167/1/tnh1og633p.pdf

Instituto Nicaragüense de Tecnología Agropecuaria (INTA). (2004). Informe Técnico Anual. Proyecto Investigación y Desarrollo, INTA Centro Norte (Matagalpa, Jinotega). 2003-2004. Managua, Nicaragua: INTA

Instituto Nicaragüense de Tecnología Agropecuaria (2017). Región VI. Centro de desarrollo tecnológico (Hugo Chavez). San Isidro, 2017. Managua, Nicaragua: INTA

Jiménez Martínez, E. S. (2009). Entomología. Managua Nicaragua: UNA.

Maes, J. M. (1998). Insectos de Nicaragua: catálogo de los insectos y artrópodos terrestres de Nicaragua. León, Nicaragua.

Mairena Vásquez, C. L. (2015). Identificación y fluctuación poblacional de insectos asociados al cultivo de la piña (Ananas comusus L. Merril.), en Ticuantepe, Nicaragua, Entre los meses de marzo a septiembre 2014. (Tesis de maestría). Recuperada de http://repositorio.una.edu.ni/3363/1/tnh1om228.pdf

Ministerio Agropecuario (MAG). (2017). Registro diario de lluvias (mm), estación Soledad de la Cruz. Municipio de Sebaco, departamento de Matagalpa. Managua, Nicaragua: MAG

Montano Núñez, R. G. y Bustamante Maradiaga, E. J. (2017). Entomofauna, diversidad $y$ fluctuación poblacional de insectos plagas y benéficos asociados al cultivo de la maracuyá (Passiflora edulis Sims), en Sébaco, Matagalpa, 2016. (Tesis de grado). Recuperada de http://repositorio.una.edu.ni/3456/1/tnh10m765.pdf

Neumann, P., y Elzen, P. (2004). La biología del pequeño escarabajo de la colmena (Aethina tumida, Coleoptera:Nitidulidae).

Nunes Zuffo, C., y Dávila Arce, M. L. (2004). Taxonomía de las Principales Familias y Subfamilias de Insectos de interés Agrícolas en Nicaragua. Estelí, Nicaragua: UCATSE.

Orozco, S.C. (1998). Cultivo del Chayote. Camoapa, Nicaragua: Centro Universitario Regional Camoapa. 
PRODES (2001). Chayote - Guía para su producción y manejo / Proyecto de Desarrollo Rural. Managua, Nicaragua: Proyecto de desarrollo rural.

Renata, A. (2002). Nueva evidencia sobre la clasificación de los Trips. San José, Costa Rica: Universidad de Costa Rica.

Reyes, R. H. (2003). EL CHAYOTE SECHIUM EDULE JACQ-SWARTZ, UNA ALTERNATIVA DE COMERCIALIZACIÓN NO TRADICIONAL. (TESIS DE GRADO) Managua, Nicaragua: Universidad Nacional Agraria.

Sáenz, M., y De La Llana, A. (1990). Entomología sistemática. Managua, Nicaragua: UNA.

Téllez Manzanares, M. del S., y Jirón Cortez, V.M. (2014). Identificación y variación poblacional de insectos asociados al cultivo de Marango (Moringa oleífera L.) en Managua, Nicaragua durante los meses de noviembre 2012 a abril 2013. (Tesis de grado). Managua, Nicaragua: Universidad Nacional Agraria.

Universidad Nacional Agraria (UNA). (2008). Guías y Formas Metodológicas de la Forma de Culminación de estudios. Managua, Nicaragua: UNA. 